\title{
Depression among diabetic patients visiting a diabetes center in Nepal
}

\author{
Durga Prasad Pahari ${ }^{1}$, Radhika Upadhyay ${ }^{2}$,Chandra Kala Sharma ${ }^{2}$ \\ ${ }^{1}$ Tribhuvan University, Institute of Medicine, Maharajgunj Medical Campus, Kathmandu. \\ ${ }^{2}$ Tribhuvan University, Institute of Medicine, Nursing Campus Maharajgunj, Kathmandu.
}

\section{Received:}

12 June 2018

\section{Revised:}

10 August 2018

\section{Accepted:}

1 October 2018

\section{${ }^{*}$ Corresponding author dppahari@iom.edu.np Tribhuvan University, Institute of Medicine, Maharajgunj Medical Campus}

\begin{abstract}
Introduction: Depression is a major co-morbidity associated with diabetes. This study aims to assess the prevalence and determine factors associated with depression among diabetic patients visiting a diabetes center in Lalitpur, Nepal.

Methods: This is a cross-sectional study of diabetic patients visiting a diabetes center in Kathmandu, Nepal. 203 diabetic patients were recruited in a period of two months. A validated and reliable Beck Depression Inventory Scale was used to identify and classify depression. Diabetes status was self-reported.Prevalence of depression, socio-demographic and diabetes related characteristics were calculated using frequency and percentages. Association was analyzed using chi-squared test. Statistical significance was determined at $\mathrm{p}<0.05$. Bivariate logistic regression was performed to identify unadjusted odds ratio with $95 \%$ CI. Then, multivariate logistic regression model was designed for those variables significant at bivariate level to calculate adjusted odds ratio with $95 \%$ CI.

Results: The prevalence of depression among diabetic patients was 34\% (Mild - 17.7\%, Moderate - 13.8\% and Severe - 2.5\%). Diabetic patients with secondary or above educational level were less than half likely to be affected by depression compared to patients with no formal school education [AOR:0.42]. Similarly, diabetic patients on insulin therapy were twice likely to be affected by depression compared to patients on oral hypoglycemic agents [AOR: 2.08] and patients having other comorbidity along with diabetes were also twice likely to be affected by depression [AOR:2.18]. Patients with stressful life events in the past were twelve times more likely to have depression compared to patients with no such events in the past [AOR: 12.33].

Conclusion: More than one third of the diabetic patients have some degree of depression. Factors such as no schooling, being on insulin therapy, having other comorbid conditions along with diabetes and stressful life events in the past among diabetic patients kept them at higher risk of depression. These factors should be focused in program for prevention and control of depression among diabetic patients in Nepal.
\end{abstract}

Key words: Diabetic patients, Depression, Beck Inventory Scale, Associated factors.

Tweetable Abstract: More than one third of diabetic patients were found to have undiagnosed depression in a care center in Nepal.

\section{Introduction}

Diabetes mellitus is increasing rapidly globally and the number of affected people is expected to reach over 366 million by the year 2030 [1]. Depression frequently occurs together with diabetes, although it is unrecognized and untreated approximately in two thirds of patients with both conditions. The course of depression in patients with both diabetes and depression is chronic and severe. More than $80 \%$ of patients with diabetes and depression experience a relapse of depressive symptoms over a 5-year period [2].

Studies have shown that diabetes was found to double the likelihood of having depression among diabetic patients when compared with non-diabetic patients [3]. Depression is found to increase the incidence of both macro vascular and micro vascular complications. It is also found to reduce quality of life and decrease compliance with medications and healthy lifestyle measures such as dietary regimes and exercise. It thereby increases health care use and expenditures and the risk of cardiovascular mortality due to poor control of hyperglycemia [4,5]. Recognition of depression is important to improve diabetic care because treatment is easily available and cost-effective [6].

There are very few evidences on prevalence and associated factors of depression among diabetic patients in Nepal. This study aimed to identify the prevalence of depression and its associated factors among diabetic patients visiting a diabetic care center in Kathmandu valley, Nepal.

\section{Methods}

This study was an institution based cross-sectional study that enrolled 203 diabetic patients in an endocrinology care centre, Lalitpur in Nepal. All the patients who visited the endocrinology care centre from 7 July to 21 July 2013 were recruited for the study purpose. Inclusion criteria for the study participants was patients with diagnosis of diabetes for more than 1 year and those aged above 20 years. Patients were excluded if they had previous history of depression, unconsciousness and with other psychotic disorders.

Beck Depression Inventory scale (Nepali translated version) of Tribhuvan University Teaching Hospital (TUTH) was used for assessing and measuring severity of depressive symptoms. 
The BDI scores and cases were categorized into "no depression (0-13)"," "milddepression (14-19)", "moderate depression (20-28)" and "severe depression (29-63)". The Nepali version of TUTH BDI scale at cut off score $13 / 14$ has sensitivity 0.93 and specificity 0.78 [7].

Structured interview schedule in Nepali version was used to gather required information from the participants through face to face interview technique at outpatients setting. BDI Score was used to explore the prevalence of depression.

Data were entered and analyzed on SPSS version 17. Status of depression, socio-demographic and diabetes related characteristics were calculated using frequency and percentages. Confidence Interval at $95 \%$ was calculated to estimate prevalence of depression among diabetic patients. Bivariate analysis was performed using cross tabulation and association was identified using chi-squared test. Statistical significance was determined at $\mathrm{p}<0.05$. Bivariate logistic regression was performed on factors with significant $p$ value to identify unadjusted odds ratio with $95 \% \mathrm{CI}$. Then, multivariable logistic regression model was used to control confounding and calculate adjusted odds ratio with $95 \%$ CI. The adjusted variables were marital status, educational level, blood sugar level, treatment modality of diabetes, presence of co-morbidity, family support and stressful life events in the past.

Research proposal was approved by research committee of Nursing Campus Maharajgunj. Official request letter was submitted to administration of diabetes thyroid and endocrinology care centre and written permission was taken to conduct research. The purpose and the procedure of the study was explained to the participants including the autonomy during the process of informed written consent with each participants.

\section{Results}

Among the 203 study participants, $60.6 \%$ of them were between age $40-60$ years and $83.3 \%$ were from inside Kathmandu valley and belonged to relatively advantage Janjati ethnic group (54.7\%). Most of the participants (89.1\%) were married and $53.2 \%$ lived in joint family. The distribution of participants according to their socio-demographic characteristics is given in Table 1.

Table 1: Socio-demographic characteristics of the study participants $(n=203)$

\begin{tabular}{lcc}
\hline Variables & Frequency & Percentage \\
\hline Age & & \\
21-60 years & 12 & 5.9 \\
41-60 years & 123 & 60.6 \\
Above 60 years & 68 & 33.5 \\
Sex & & \\
Male & 102 & 50.2 \\
Female & 101 & 49.8 \\
Address & & \\
Inside Kathmandu Valley & 169 & 83.3 \\
Outside Kathmandu Valley & 34 & 16.7 \\
\hline
\end{tabular}

\begin{tabular}{|c|c|c|}
\hline Variables & Frequency & Percentage \\
\hline \multicolumn{3}{|l|}{ Ethnicity } \\
\hline Dalit & 3 & 1.5 \\
\hline $\begin{array}{l}\text { Disadvantaged Janajatis, Madhesi and } \\
\text { religious minorities }\end{array}$ & 17 & 8.4 \\
\hline Relatively advantaged Janajatis & 111 & 54.7 \\
\hline Upper caste group & 72 & 35.5 \\
\hline \multicolumn{3}{|l|}{ Marital Status } \\
\hline Married & 181 & 89.1 \\
\hline Unmarried & 6 & 3.0 \\
\hline Widow & 16 & 7.9 \\
\hline \multicolumn{3}{|l|}{ Family type } \\
\hline Nuclear & 95 & 46.8 \\
\hline Joint & 108 & 53.2 \\
\hline \multicolumn{3}{|l|}{ Occupation } \\
\hline Retired & 57 & 28.1 \\
\hline House wife & 53 & 26.1 \\
\hline Business & 45 & 22.2 \\
\hline Services & 37 & 18.2 \\
\hline Others & 11 & 5.4 \\
\hline \multicolumn{3}{|l|}{ Economic Status } \\
\hline Rich & 112 & 55.2 \\
\hline Average & 78 & 38.4 \\
\hline Poor & 13 & 6.4 \\
\hline \multicolumn{3}{|l|}{ Educational Level } \\
\hline Illiterate & 43 & 21.2 \\
\hline Literate & 5 & 2.4 \\
\hline Primary & 17 & 8.4 \\
\hline Secondary & 24 & 11.8 \\
\hline SLC & 27 & 13.3 \\
\hline Intermediate & 32 & 15.8 \\
\hline Bachelors & 39 & 19.2 \\
\hline Masters & 16 & 7.9 \\
\hline
\end{tabular}

The overall prevalence of depression with 95\% CI was 34\% (27.5\%41.0\%). The level of depression with $95 \%$ CI was mild $17.7 \%$ $(12.7 \%-23.7 \%)$, moderate $13.8 \%(9.4 \%-19.3 \%)$ and severe $2.5 \%$ (0.8\%-5.7\%). (Table 2)

Table 2: Depression among diabetic patients $(n=203)$

\begin{tabular}{lcc}
\hline $\begin{array}{l}\text { Depression among diabetic } \\
\text { patients }\end{array}$ & Frequency & $\begin{array}{c}\text { Percentage } \\
(\mathbf{9 5 \%} \mathbf{C I})\end{array}$ \\
\hline $\begin{array}{l}\text { Prevalence of Depression } \\
\text { No depression }\end{array}$ & 134 & $66.0(59.0-72.5)$ \\
Depression & 69 & $34.0(27.5-41.0)$ \\
Level of depression & & \\
No depression (0-13) & 134 & $66(59.0-72.5)$ \\
Mild depression (14-19) & 36 & $17.7(12.7-23.7)$ \\
Moderate depression (20-28) & 28 & $13.8(9.4-19.3)$ \\
Severe depression (29-63) & 5 & $2.5(0.8-5.7)$ \\
Total & 203 & 100.0 \\
\hline
\end{tabular}

Among the socio-demographic characteristics, depression was associated with marital status and educational level of the study participants. (Table 3) 
Table 3: Association of socio-demographic characteristics with depression among diabetic patients $(n=203)$

\begin{tabular}{|c|c|c|c|}
\hline Characteristics & $\begin{array}{c}\text { No Depression } \\
\mathbf{n}(\%)\end{array}$ & $\begin{array}{c}\text { Depression } \\
\text { n (\%) }\end{array}$ & $P$ value \\
\hline $\begin{array}{l}\text { Sex } \\
\text { Male } \\
\text { Female }\end{array}$ & $\begin{array}{l}70(68.6 \%) \\
64(63.4 \%)\end{array}$ & $\begin{array}{l}32(31.4 \%) \\
37(36.6 \%)\end{array}$ & 0.428 \\
\hline $\begin{array}{l}\text { Age group } \\
20-40 \text { years } \\
41-50 \text { years } \\
51-60 \text { years } \\
\text { Above } 60 \text { years }\end{array}$ & $\begin{array}{c}8(66.7 \%) \\
39(78 \%) \\
45(61.6 \%) \\
42(61.8 \%)\end{array}$ & $\begin{array}{c}4(33.3 \%) \\
11(22.0 \%) \\
28(38.4 \%) \\
26(38.2 \%)\end{array}$ & 0.223 \\
\hline $\begin{array}{l}\text { Address } \\
\text { Inside Kathmandu } \\
\text { valley } \\
\text { Outside Kathmandu } \\
\text { valley }\end{array}$ & $20(58.8 \%)$ & $14(41.2 \%)$ & 0.332 \\
\hline $\begin{array}{l}\text { Ethnicity } \\
\text { Disadvantaged Jan- } \\
\text { jatis and Dalits } \\
\text { Relatively advan- } \\
\text { taged Janajatis } \\
\text { Upper caste group }\end{array}$ & $\begin{array}{l}76(68.5 \%) \\
44(61.1 \%)\end{array}$ & $\begin{array}{l}6(30.0 \%) \\
35(31.5 \%) \\
28(38.9 \%)\end{array}$ & 0.545 \\
\hline $\begin{array}{l}\text { Marital status } \\
\text { Married } \\
\text { Unmarried or wid- } \\
\text { ow/widower }\end{array}$ & $\begin{array}{c}124(68.5 \%) \\
10(45 \%)\end{array}$ & $\begin{array}{c}57(31.5 \%) \\
12(55 \%)\end{array}$ & $0.031^{*}$ \\
\hline $\begin{array}{l}\text { Occupation } \\
\text { Services } \\
\text { Business } \\
\text { House wife } \\
\text { Retired } \\
\text { Others (Agriculture } \\
\text { and unemployed) }\end{array}$ & $\begin{array}{c}26(70.3 \%) \\
31(68.9 \%) \\
37(69.8 \%) \\
33(57.9 \%) \\
5(45 \%)\end{array}$ & $\begin{array}{c}11(29.7 \%) \\
14(31.1 \%) \\
16(30.2 \%) \\
24(42.1 \%) \\
6(55 \%)\end{array}$ & 0.354 \\
\hline $\begin{array}{l}\text { Family type } \\
\text { Nuclear } \\
\text { Joint }\end{array}$ & $\begin{array}{l}63(66.3 \%) \\
71(65.7 \%)\end{array}$ & $\begin{array}{l}32(33.7 \%) \\
37(34.3 \%)\end{array}$ & 0.930 \\
\hline $\begin{array}{l}\text { Economic status } \\
\text { Average } \\
\text { Poor } \\
\text { Rich }\end{array}$ & $\begin{array}{c}47(60.3 \%) \\
6(46.2 \%) \\
81(72.3 \%)\end{array}$ & $\begin{array}{c}31(39.7 \%) \\
7(53.8 \%) \\
31(27.7 \%)\end{array}$ & 0.066 \\
\hline $\begin{array}{l}\text { Education level } \\
\text { No schooling } \\
\text { Primary } \\
\text { Secondary or above }\end{array}$ & $\begin{array}{c}26(54 \%) \\
9(52.9 \%) \\
99(71.7 \%)\end{array}$ & $\begin{array}{c}22(46 \%) \\
8(47.1 \%) \\
39(28.3 \%)\end{array}$ & $0.042^{*}$ \\
\hline
\end{tabular}

Among the diabetes related characteristics, blood sugar level, treatment modality of diabetes, presence of co-morbid diseases, family support and stressful life events were significantly associated with depression. (Table 4)
Table 4: Association of diabetes related characteristics and depression $(n=203)$

\begin{tabular}{|c|c|c|c|}
\hline Characteristics & $\begin{array}{l}\text { No Depres- } \\
\text { sion n (\%) }\end{array}$ & $\begin{array}{l}\text { Depression } \\
\text { n }(\%)\end{array}$ & $P$ value \\
\hline $\begin{array}{l}\text { Duration of diabetes } \\
1-3 \text { years } \\
4-9 \text { years } \\
10 \text { years or more }\end{array}$ & $\begin{array}{l}44(74.6 \%) \\
30(56.5 \%) \\
60(65.6 \%)\end{array}$ & $\begin{array}{l}15(25.4 \%) \\
23(43.5 \%) \\
31(34.4 \%)\end{array}$ & 0.133 \\
\hline $\begin{array}{l}\text { PP sugar level } \\
\text { Below } 200 \mathrm{mg} / \mathrm{dl} \\
200 \mathrm{mg} / \mathrm{dl} \text { and above }\end{array}$ & $\begin{array}{l}93(72.1 \%) \\
41(55.4 \%)\end{array}$ & $\begin{array}{l}36(27.9 \%) \\
33(44.6 \%)\end{array}$ & $0.016^{*}$ \\
\hline $\begin{array}{l}\text { Fast blood sugar level } \\
\text { Below } 125 \mathrm{mg} / \mathrm{dl} \\
125 \mathrm{mg} / \mathrm{dl} \text { and above }\end{array}$ & $\begin{array}{l}74(72.5 \%) \\
60(59.4 \%)\end{array}$ & $\begin{array}{l}28(27.5 \%) \\
41(40.6 \%)\end{array}$ & $0.048^{*}$ \\
\hline $\begin{array}{l}\text { BMI } \\
16-18 \\
19-25 \\
26-30 \\
>30\end{array}$ & $\begin{array}{l}4(80 \%) \\
79(68.1 \%) \\
45(64.3 \%) \\
6(50 \%)\end{array}$ & $\begin{array}{l}1(20 \%) \\
37(31.9 \%) \\
25(35.7 \%) \\
6(50 \%)\end{array}$ & 0.546 \\
\hline $\begin{array}{l}\text { HBA1C level } \\
4.5-7 \% \\
>7 \%\end{array}$ & $\begin{array}{l}43(63.2 \%) \\
91(67.4 \%)\end{array}$ & $\begin{array}{l}25(36.8 \%) \\
44(32.6 \%)\end{array}$ & 0.055 \\
\hline $\begin{array}{l}\text { Exercise duration } \\
\text { (hrs/week) } \\
0 \\
0.5-3 \\
4-10 \\
11-16\end{array}$ & $\begin{array}{l}29(59.2 \%) \\
41(66.1 \%) \\
59(69.4 \%) \\
5(71.4 \%)\end{array}$ & $\begin{array}{l}20(40.8 \%) \\
21(33.9 \%) \\
26(30.6 \%) \\
2(28.6 \%)\end{array}$ & 0.671 \\
\hline $\begin{array}{l}\text { Treatment modality } \\
\text { of diabetes } \\
\text { Oral Hypoglycemic } \\
\text { agents } \\
\text { Insulin therapy }\end{array}$ & $\begin{array}{l}86(72.9 \%) \\
48(56.5 \%)\end{array}$ & $\begin{array}{l}32(27.1 \%) \\
37(43.5 \%)\end{array}$ & $0.014^{\star}$ \\
\hline $\begin{array}{l}\text { Presence of co-mor- } \\
\text { bid diseases } \\
\text { No any co morbid } \\
\text { disease } \\
\text { Having co-morbid } \\
\text { disease/s }\end{array}$ & $\begin{array}{l}34(87.2 \%) \\
100(61 \%)\end{array}$ & $\begin{array}{l}5(12.8 \%) \\
64(39 \%)\end{array}$ & $0.001^{\star}$ \\
\hline $\begin{array}{l}\text { Family support } \\
\text { Yes } \\
\text { No }\end{array}$ & $\begin{array}{l}133(67.9 \%) \\
1(14.3 \%)\end{array}$ & $\begin{array}{l}63(32.1 \%) \\
6(85.7 \%)\end{array}$ & $0.003^{*}$ \\
\hline $\begin{array}{l}\text { Stressful life events } \\
\text { Yes } \\
\text { No }\end{array}$ & $\begin{array}{l}1(12.5 \%) \\
133(68.2 \%)\end{array}$ & $\begin{array}{l}7(87.5 \%) \\
62(31.8 \%)\end{array}$ & $0.001^{\star}$ \\
\hline $\begin{array}{l}\text { Family history } \\
\text { Positive } \\
\text { Negative }\end{array}$ & $\begin{array}{l}3(37.5 \%) \\
131(67.2)\end{array}$ & $\begin{array}{l}5(62.5 \%) \\
64(32.8 \%) \\
\end{array}$ & 0.082 \\
\hline
\end{tabular}

Among the socio-demographic and diabetes related factors, the factors which showed significant association with depression (marital status, educational level, blood sugar level, treatment modality of diabetes, presence of co-morbid diseases, family support and stressful life events) were taken into logistic regression analysis and adjusted and unadjusted odds ratio with 95\% CI were calculated. After controlling confounding, secondary and above schooling 
level compared to no schooling [AOR: 0.42 (0.19-0.90)], patients in insulin therapy compared to hypoglycemic agents [AOR: 2.08 (1.06-4.05)], patients having other comorbid diseases compared to not having them [AOR: 2.18 (1.13-4.22)] and patients having stressful life events compared to not having them in the past [AOR: 12.33 (1.23-123.27)] were found statistically significant with depression in the final model. (Table 5)

Table 5: Factors affecting depression among diabetic patients from regression analysis $(n=203)$

\begin{tabular}{|c|c|c|c|c|}
\hline Characteristics & $\begin{array}{c}\text { Unadjusted } \\
\text { odds ratio } \\
(95 \% \mathrm{CI})\end{array}$ & P value & $\begin{array}{c}\text { Adjusted } \\
\text { odds ratio } \\
(95 \% \mathrm{CI})\end{array}$ & $P$ value \\
\hline \multicolumn{5}{|l|}{ Marital status } \\
\hline Married & 1 & & 1 & \\
\hline $\begin{array}{l}\text { Unmarried } \\
\text { or Widow/ } \\
\text { Widower }\end{array}$ & $\begin{array}{l}2.61(1.06- \\
\quad 6.39)\end{array}$ & $0.036^{*}$ & $\begin{array}{c}1.39(0.46- \\
4.15)\end{array}$ & 0.554 \\
\hline \multicolumn{5}{|l|}{ Education Level } \\
\hline No Schooling & 1 & & 1 & \\
\hline Primary & $\begin{array}{c}1.05(0.34- \\
3.81)\end{array}$ & 0.931 & $\begin{array}{l}0.76(0.20- \\
2.85)\end{array}$ & 0.695 \\
\hline Secondary & $\begin{array}{l}0.46(0.23- \\
0.91)\end{array}$ & $0.027^{\star}$ & $\begin{array}{c}0.42(0.19- \\
0.90)\end{array}$ & $0.026^{*}$ \\
\hline \multicolumn{5}{|l|}{ PP sugar level } \\
\hline Below 200 mg/dl & 1 & & & \\
\hline $200 \mathrm{mg} / \mathrm{dl}$ and above & $\begin{array}{l}2.07(1.14- \\
3.78)\end{array}$ & $0.017^{\star}$ & $\begin{array}{c}1.77(0.87- \\
3.59)\end{array}$ & 0.111 \\
\hline \multicolumn{5}{|l|}{$\begin{array}{l}\text { Fast Blood sugar } \\
\text { level }\end{array}$} \\
\hline Below 125 mg/dl & 1 & & 1 & \\
\hline $125 \mathrm{mg} / \mathrm{dl}$ and above & $\begin{array}{c}1.80(1.002- \\
3.25)\end{array}$ & $0.049^{*}$ & $\begin{array}{c}1.25 \\
(0.62-2.52)\end{array}$ & 0.526 \\
\hline \multicolumn{5}{|l|}{$\begin{array}{l}\text { Treatment modality } \\
\text { of diabetes }\end{array}$} \\
\hline $\begin{array}{l}\text { Oral hypoglycemic } \\
\text { agents }\end{array}$ & 1 & & 1 & \\
\hline Insulin therapy & $\begin{array}{c}2.07(1.14- \\
3.73)\end{array}$ & $0.409^{*}$ & $\begin{array}{l}2.08(1.06- \\
4.05)\end{array}$ & $0.032^{*}$ \\
\hline \multicolumn{5}{|l|}{$\begin{array}{l}\text { Presence of co-mor- } \\
\text { bid diseases }\end{array}$} \\
\hline No & 1 & & 1 & \\
\hline Yes & $\begin{array}{c}2.10(1.16- \\
3.79)\end{array}$ & $0.014^{*}$ & $\begin{array}{l}2.18(1.13- \\
\quad 4.22)\end{array}$ & $0.020^{*}$ \\
\hline \multicolumn{5}{|l|}{ Family support } \\
\hline Yes & 1 & & 1 & \\
\hline No & $\begin{array}{c}12.66(1.49- \\
107.45)\end{array}$ & $0.020^{\star}$ & $\begin{array}{l}9.41(0.95- \\
92.72)\end{array}$ & 0.055 \\
\hline \multicolumn{5}{|l|}{ Stressful life events } \\
\hline Yes & $\begin{array}{c}15.01(1.80- \\
124.70)\end{array}$ & $0.012^{*}$ & $\begin{array}{l}12.33(1.23 \\
-123.27)\end{array}$ & $0.032^{*}$ \\
\hline No & 1 & & 1 & \\
\hline
\end{tabular}

\section{Discussion}

The study showed quite high prevalence of depression among Nepali diabetic patients. The overall prevalence was $34 \%$ in which $17.7 \%$ were mild, $13.8 \%$ were moderate and $2.5 \%$ were severely depressed. Similar studies reported slightly higher prevalence from
South India (37.5\%), North India (41\%) and Saudi Arabia (49.6\%) respectively [8-10].

This study at bivariate analysis found among socio-demographic characteristics, marital status and education level were significantly associated with depression while in multivariable logistic regression analysis only secondary and above level education was found to have significantly low depression compared to no schooling. A study in Saudi Arabia also showed marital status significantly associated with depression [10] while a study done in Nigeria showing contrasting findings to this study that income and family size associated with depression among diabetic patients [11]. This may be because of not enough power of this study to detect the association. Previous studies showed comorbid conditions and increased fasting glucose level were significantly associated with depression $[8,12]$. Also, family support influences the psychosocial outcomes in diabetes patients [13]. A study in Ethiopia also showed similar findings that negative life events and poor social support were significantly associated with depression among diabetic patients [14]. Our findings on the association of treatment modality with depression is supported by a previous study which showed oral hypoglycemic therapy is potentially safer and caters less risk of depression in comparison to intensive management using multiple daily injections [15].

This study was limited to patients visiting a privately run care center in Kathmandu valley so these patients may not represent all diabetic patients in Nepal. Despite this limitation, the magnitude and associated factors identified by this study might be useful for programs to control depression among diabetes patients in Nepal.

\section{Conclusion}

More than one third of the diabetic patients had some degree of undiagnosed depression. Factors like no schooling, being on insulin therapy, having comorbid conditions and stressful life events increased the chances of developing depressive symptoms. Mental health counselling and education is an immediate priority for those with depressive symptoms and can benefit from regular glucose monitoring and screening of depression.

\section{Acknowledgements}

We extend our thanks to Dr Amit Shakya, Director of diabetes, thyroid and endocrinology care center, Lalitpur and his team for their support in the process of data collection.

\section{Conflict of interest}

None declared.

\section{References}

1. Wild S, Roglic G, Green A, Sicree R, King H. Global prevalence of diabetes: estimates for the year 2000 and projections for 2030. Diabetes Care. 2004;27(5):104753.

2. Lin EH, Rutter CM, Katon W, Heckbert SR, Ciechanowski P, Oliver MM, et al. depression and advanced complications of diabetes: a prospective cohort study. Diabetes Care. 2010;33(2):264-9.

3. Thomas J, Jones G, Scarinci I, Brantley P. A descriptive and comparative study of the prevalence of depressive and anxiety disorders in low-income adults with type 2 diabetes and other chronic illnesses. Diabetes Care. 2003;26(8):2311-7.

4. de Groot M, Anderson R, Freedland KE, Clouse RE, Lustman PJ. Association 
of depression and diabetes complications: a meta-analysis. Psychosom Med. 2001;63(4):619-30.

5. Rahman M, Rahman MA, Flora MS, Rakibuz-Zaman M. Depression and associated factors in diabetic patients attending an Urban hospital of Bangladesh. Int J Collab Res Intern Med Public Health 2011;3:65-76.

6. Lustman PJ, Griffith LS, Clouse RE, Freedland KE, Eisen SA, Rubin EH, et al. Effects of nortriptyline on depression and glycemic control in diabetes: results of a double-blind, placebo-controlled trial. Psychosom Med. 1997;59(3):241-50.

7. Kohrt BA, Kunj RD, Koiraka NR, Sharma VD, Nepal MK. Validation of a Nepali version of the Beck Depression Inventory. Nepalese Journal of Psychiatry 2002;2

(4):123-130.

8. Aminu AS, Chandrasekaran V, Nair S. Depression among Patients with Diabetes: A Community-based Study in South India. J Med Sci 2017;37(6):237-244.

9. Raval A, Dhanaraj E, Bhansali A, Grover S, Tiwari P. Prevalence and determinants of depression in type 2 diabetes patients in a tertiary care centre. Indian J Med Res. 2010;132:195-200.

10. El Mahalli AA. Prevalence and predictors of depression among type 2 diabetes mellitus outpatients in eastern province, Saudi Arabia. Int J Health Sci (Qassim). 2015;9(2):119-26.

11. James BO, Omoaregba JO, Eze G, Morakinyo O. Depression among patients with diabetes mellitus in a Nigerian teaching hospital. South African Journal of Psychiatry. 2010;16(2):61-64.

12. Khuwaja AK, Lalani S, Dhanani R, Azam IS, Rafique G, White F. Anxiety and depression among outpatients with type 2 diabetes: A multi-centre study of prevalence and associated factors. Diabetol Metab Syndr. 2010;20(2):72.

13. Whittemore R, Kanner S, Singleton S, Hamrin V, Chiu J, Grey M. Correlates of depressive symptoms in adolescents with type 1 diabetes. Pediatr Diabetes. 2002;3(3):135-43.

14. Habtewold TD, Alemu SM, Haile YG. Sociodemographic, clinical, and psychosocial factors associated with depression among type 2 diabetic outpatients in Black Lion General Specialized Hospital, Addis Ababa, Ethiopia: a cross-sectional study. BMC Psychiatry. 2016;15;16:103.

15. Weinzimer SA, Ahern JH, Doyle EA, Vincent MR, Dziura J, Steffen AT, et al. Persistence of benefits of continuous subcutaneous insulin infusion in very young children with type 1 diabetes: a follow-up report. Pediatrics. 2004;114(6):1601-5. 